\title{
Two-dimension Coherent DOA Estimation for Uniform Planar Array via Reconstructing Toeplitz Matrix
}

\author{
Shihong Chen ${ }^{1, a^{*}}$, Xiaofei Zhang ${ }^{1, b}$ and Chi Jiang ${ }^{1, c}$ \\ ${ }^{1}$ (College of Electronic Information Engineering, Nanjing University of Aeronautics and \\ Astronautics, Nanjing 210016, China) \\ acch041230104@nuaa.edu.cn, bzhangxiaofei@nuaa.edu.cn, jiangchi1991_nuaa@163.com
}

Keywords: coherent signal; Direction of arrival (DOA) estimation; uniform planar array Abstract. The problem of two-dimension coherent direction of arrival (DOA) for uniform planar array is investigated in this paper. The signals propagate over multipath channel, which thus leads to the coherent source. The traditional two-dimension direction of arrival (2D-DOA) estimation algorithm fails to work for the coherent source. In this paper, we will propose a coherent DOA estimation algorithm for uniform planar array, which uses the characteristic of Toeplitz matrix to restructure the covariance matrix, and use rotational invariance technique to realize the 2D-DOA estimation. The validity and performance of the algorithm can be verified by simulations in this paper.

\section{Introduction}

Direction-of-arrival (DOA) estimation is a fundamental problem in array signal processing and has been widely used in many fields, such as sonar, radar, wireless communication [1-3]. In recent years, with the rise of two-dimensional (2D) parameter estimation, the research and application of 2D-DOA estimation have won great attention in array signal processing [4-11]. This problem is usually considered with rectangular array, two parallel uniform linear array, and L-shape array, etc. Also, many algorithms have been applied to 2D-DOA estimation, which include 2D Multiple Signal Classification (MUSIC) algorithm [4], 2D Estimation of Signal Parameters via Rotational Invariance Technique (ESPRIT) algorithm [5-6], 2D Propagator Method (PM) algorithm [7-8], maximum likelihood method [9], Parallel Factor (PARAFAC) algorithm [10] and high order cumulant method [11], etc.

However, under the influence of environment, the signal emitted by the source, will propagate through multipath, thus leading to the coherent signal sources. The traditional DOA estimation methods assume that the signals are independent statistically, but we should consider the rank loss of the covariance matrix of the coherent sources. If using the traditional method, we will directly obtain inaccurate or even error angles. Therefore, it is valuable to study the effective coherent signal processing algorithm. The focus of DOA estimation of coherent signals is how to restore the rank of the covariance matrix, which called decoherence, and then use the conventional method to estimate DOA. The pretreatment of decoherence can be divided into two categories: spatial smoothing algorithm [12] and time smoothing algorithm [13]. The essence of spatial smoothing algorithm is to recover matrix rank, but the dimension of the matrix is less than the revised dimension of the original matrix. That is to say, the spatial smoothing algorithm is used by reducing the degree of freedom.

In this paper, a new algorithm of 2D-DOA estimation for the coherent sources is proposed in the uniform planar array. The algorithm reconstructs a special antenna array model based on the Toeplitz-like matrix whose rank is only related to the DOA of signals and cannot be affected by the coherency between them [14]. Furthermore, the ESPRIT algorithm will be used to estimate the 2D-DOA.

This paper consists of 5 sections: the first section is introduction; the second section analyzes the signal model; the third section proposes an ESPRIT algorithm via reconstructing Toeplitz-like matrix; the fourth section conducts simulation; and the last section is the conclusion.

Notion: $(\bullet)^{T},(\bullet)^{H},(\bullet)^{-1}$ and $(\bullet)^{+}$denote transpose, conjugate-transpose, inverse, pseudo-inverse operations, respectively. $E[\bullet],\|\|_{F}$ and $O[\square$ are expectation operator, Frobenius 
norm and complexity, respectively. $\operatorname{diag}(\mathbf{v})$ stands for diagonal matrix whose diagonal element is a vector v. angle $(\bullet)$ is to get the phase angle. $\hat{x}$ represents estimation of exact value $x . \otimes, 0$ and $\oplus$ stand for the Kronecker product, Khatri-Rao product and Hadamard product, respectively. bold lowercase letters are vectors and bold capital letters are matrixes.

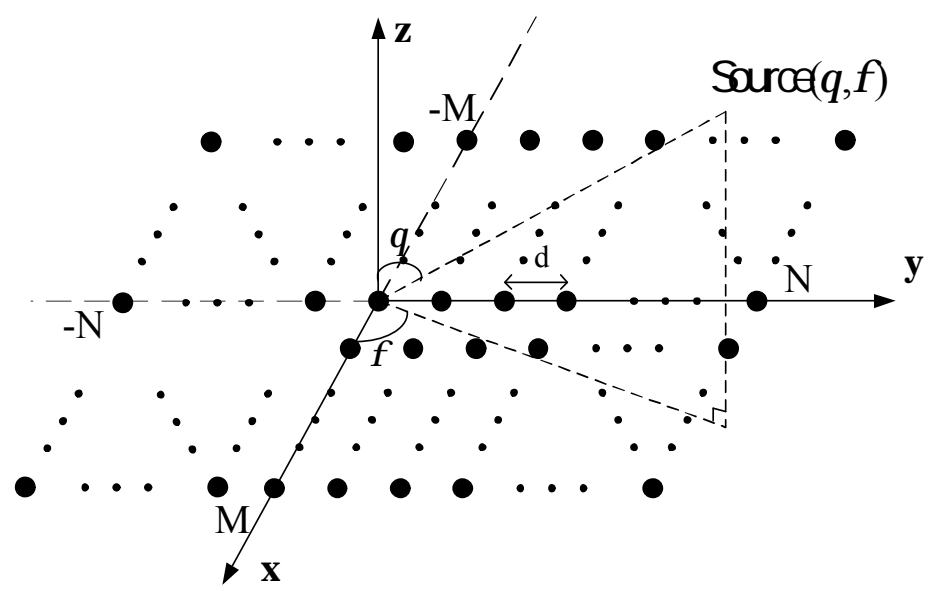

Fig. 1 The structure of uniform planar array ${ }^{[1]}$

\section{Signal Model}

Fig.1 shows a uniform planar array which has $(2 M+1) \times(2 N+1)$ sensors, and the space between adjacent elements is $d$. Allowing for the far field signal with distant source, the signal arriving at the array can be considered as parallel wave. Suppose that the noise and signal are mutually independent and it is an addictive independently and identically distributed Gaussian process. Assume that there are $K$ signal sources, where the first $Q$ signals are mutually coherent and others are uncorrelated and independent of the first $Q$ signals, and the elevation angle and azimuth angle of $i$ th source are $\theta_{i}$ and $\phi_{i}$. The received signal of the $(m, n)$ element can be expressed as

$$
\begin{aligned}
x_{m n}(t) & =\sum_{k=1}^{K} s_{k}(t) e^{-j 2 \pi d m \sin \theta_{k} \cos \phi_{k} / \lambda} e^{-j 2 \pi d n \sin \theta_{k} \sin \phi_{k} / \lambda}+n_{m n}(t) \\
& =s_{1}(t) \sum_{k=1}^{Q} \beta_{k} e^{-j 2 \pi d m \sin \theta_{k} \cos \phi_{k} / \lambda} e^{-j 2 \pi d n \sin \theta_{k} \sin \phi_{k} / \lambda} \\
& +\sum_{k=Q+1}^{K} s_{k}(t) e^{-j 2 \pi d m \sin \theta_{k} \cos \phi_{k} / \lambda} e^{-j 2 \pi d n \sin \theta_{k} \sin \phi_{k} / \lambda}+n_{m n}(t)
\end{aligned}
$$

where $s_{k}(t)$ is the $k$ th source, $n_{m n}(t)$ is the additive white Gaussian noise, and $\beta_{k}$ represents coherence coefficient. Then the received signal is given by 


$$
\begin{aligned}
\mathbf{X}(t) & =\left[\begin{array}{ccccc}
x_{-M-N}(t) & \mathrm{L} & x_{0-N}(t) & \mathrm{L} & x_{M-N}(t) \\
\mathrm{M} & \mathrm{O} & \mathrm{M} & \mathrm{O} & \mathrm{M} \\
x_{-M 0}(t) & \mathrm{L} & x_{00}(t) & \mathrm{L} & x_{M 0}(t) \\
\mathrm{M} & \mathrm{O} & \mathrm{M} & \mathrm{O} & \mathrm{M} \\
x_{-M N}(t) & \mathrm{L} & x_{0 N}(t) & \mathrm{L} & x_{M N}(t)
\end{array}\right]^{T}=\mathbf{A s}(t)+\mathbf{n}(t) \\
& =\left[\begin{array}{c}
A_{x} \Psi^{-N} \\
\mathrm{M} \\
A_{x} \Psi^{0} \\
A_{x} \Psi^{1} \\
\mathrm{M} \\
A_{x} \Psi^{N}
\end{array}\right] \mathbf{s}(t)+\mathbf{n}(t)=\left(A_{y} \mathrm{O} A_{x}\right) \mathbf{s}(t)+\mathbf{n}(t)
\end{aligned}
$$

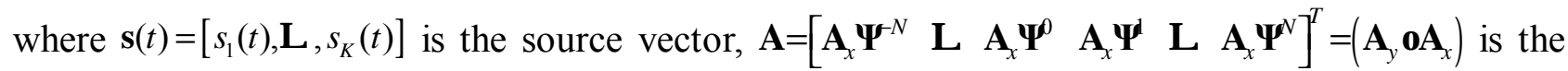
direction matrix, $\mathbf{A}_{y}=\left[\mathbf{a}_{y}\left(\theta_{1} \phi_{1}\right), \mathbf{a}_{y}\left(\theta_{2} \phi_{2}\right), \mathrm{L}, \mathbf{a}_{y}\left(\theta_{K} \phi_{K}\right)\right], \mathbf{A}_{x}=\left[\mathbf{a}_{x}\left(\theta_{1} \phi_{1}\right), \mathbf{a}_{x}\left(\theta_{2} \phi_{2}\right), \mathrm{L}, \mathbf{a}_{x}\left(\theta_{K} \phi_{K}\right)\right]$,

$\mathbf{a}_{y}\left(\theta_{k} \phi_{k}\right)=\left[e^{j 2 \pi N d \sin \theta_{k} \sin \phi_{k} / \lambda}, \mathrm{L}, e^{j 2 \pi d \sin \theta_{k} \sin \phi_{k} / \lambda}, 1, e^{-j 2 \pi d \sin \theta_{k} \sin \phi_{k} / \lambda}, \mathrm{L}, e^{-j 2 \pi N d \sin \theta_{k} \sin \phi_{k} / \lambda}\right]^{T}$

$\mathbf{a}_{x}\left(\theta_{k} \phi_{k}\right)=\left[e^{j 2 \pi M d \sin \theta_{k} \cos \phi_{k} / \lambda}, \mathrm{L}, e^{j 2 \pi d \sin \theta_{k} \cos \phi_{k} / \lambda}, 1, e^{-j 2 \pi d \sin \theta_{k} \cos \phi_{k} / \lambda}, \mathrm{L}, e^{-j 2 \pi M d \sin \theta_{k} \cos \phi_{k} / \lambda}\right]^{T} \quad, \quad$ and $\boldsymbol{\Psi}=\operatorname{diag}\left(e^{-j 2 \pi d \sin \theta_{1} \sin \phi_{1} / \lambda}, e^{-j 2 \pi d \sin \theta_{2} \sin \phi_{2} / \lambda}, \ldots, e^{-j 2 \pi d \sin \theta_{K} \sin \phi_{K} / \lambda}\right)$.

Therefore, the covariance matrix can be given as $\mathbf{R}_{\mathrm{XX}}=E\left[\mathbf{X}(t) \mathbf{X}(t)^{H}\right]$, and any element of the covariance matrix can be expressed as

$$
\begin{aligned}
r(m n, p q) & =\sum_{k=1}^{K} d_{m n, k} e^{j 2 \pi d p \sin \theta_{k} \cos \phi_{k} / \lambda} e^{j 2 \pi d q \sin \theta_{k} \sin \phi_{k} / \lambda} \\
& +\sigma^{2} \delta_{m n, p q}(m, p=-M, \mathrm{~L} 0, \mathrm{~L} M ; n, q=-N, \mathrm{~L} 0, \mathrm{~L} N)
\end{aligned}
$$

where

$$
\begin{aligned}
& d_{m n, k}=\left\{\begin{array}{c}
P_{1,1} \beta_{k}^{*} \sum_{I=1}^{Q} \beta_{l} e^{-j 2 \pi d m \sin \theta_{k} \cos \phi_{k} / \lambda} e^{-j 2 \pi d n \sin \theta_{k} \sin \phi_{k} / \lambda}, k=1, \mathrm{~L}, Q \\
P_{k, k} e^{-j 2 \pi d m \sin \theta_{k} \cos \phi_{k} / \lambda} e^{-j 2 \pi d n \sin \theta_{k} \sin \phi_{k} / \lambda}, k=Q+1, \mathrm{~L}, K
\end{array}\right. \\
& P_{l, k}=E\left[s_{l}(t) s_{k}^{*}(t)\right](l, k=1, Q+1, \mathrm{~L}, K) \text { and } \sigma^{2} \text { is noise power. }
\end{aligned}
$$

\section{An ESPRIT Algorithm via Reconstructing Toeplitz-like Matrix}

We can construct Toeplitz-like matrix

$$
\mathbf{R}(m n)=\left[\begin{array}{ccccccccc}
r(m n, 00) & r(m n, 01) & \mathrm{L} & r(m n, 0 N) & r(m n, 10) & \mathrm{L} & r(m n, 1 N) & \mathrm{L} & r(m n, M N) \\
r(m n, 0-1) & r(m n, 00) & \mathrm{L} & r(m n, 0(N-1)) & r(m n, 1-1) & \mathrm{L} & r(m n, 1(N-1)) & \mathrm{L} & r(m n, M(N-1)) \\
\mathrm{M} & \mathrm{M} & \mathrm{O} & \mathrm{M} & \mathrm{M} & \mathrm{O} & \mathrm{M} & \mathrm{O} & \mathrm{M} \\
r(m n, 0-N) & r(m n, 0(1-N)) & \mathrm{L} & r(m n, 00) & r(m n, 1-N) & \mathrm{L} & r(m n, 10) & \mathrm{L} & r(m n, M 0) \\
r(m n,-10) & r(m n,-11) & \mathrm{L} & r(m n,-1 N) & r(m n, 00) & \mathrm{L} & r(0 N) & \mathrm{L} & r(m n,(M-1) N) \\
\mathrm{M} & \mathrm{M} & \mathrm{O} & \mathrm{M} & \mathrm{M} & \mathrm{O} & \mathrm{M} & \mathrm{O} & \mathrm{M} \\
r(m n,-1-N) & r(m n,-1(1-N)) & \mathrm{L} & r(m n,-10) & r(m n, 0-N) & \mathrm{L} & r(m n, 00) & \mathrm{L} & r(m n,(M-1) 0) \\
\mathrm{M} & \mathrm{M} & \mathrm{O} & \mathrm{M} & \mathrm{M} & \mathrm{O} & \mathrm{M} & \mathrm{O} & \mathrm{M} \\
r(m n,-M-N) & r(m n,-M(1-N)) & \mathrm{L} & r(m n,-M 0) & r(m n,(1-M)-N) & \mathrm{L} & r(m n,(1-M) 0) & \mathrm{L} & r(m n, 00)
\end{array}\right]
$$


where $\mathbf{A}_{r}=\mathbf{A}_{r y} \mathbf{O} \mathbf{A}_{r x}, \mathbf{A}_{r x}=\left[\mathbf{a}_{r x}\left(\theta_{1} \phi_{1}\right), \mathbf{a}_{r x}\left(\theta_{2} \phi_{2}\right), \mathrm{L}, \mathbf{a}_{r x}\left(\theta_{K} \phi_{K}\right)\right], \mathbf{A}_{r y}=\left[\mathbf{a}_{r y}\left(\theta_{1} \phi_{1}\right), \mathbf{a}_{r y}\left(\theta_{2} \phi_{2}\right), \mathrm{L}, \mathbf{a}_{r y}\left(\theta_{K} \phi_{K}\right)\right]$, $\mathbf{a}_{r x}\left(\theta_{k} \phi_{k}\right)=\left[1, e^{-j 2 \pi d \sin \theta_{k} \cos \phi_{k} / \lambda}, \mathrm{L}, e^{-j 2 \pi N d \sin \theta_{k} \cos \phi_{k} / \lambda}\right]^{T}, \mathbf{a}_{r y}\left(\theta_{k} \phi_{k}\right)=\left[1, e^{-j 2 \pi d \sin \theta_{k} \sin \phi_{k} / \lambda}, \mathrm{L}, e^{-j 2 \pi N d \sin \theta_{k} \sin \phi_{k} / \lambda}\right]^{T}$ and $\mathbf{D}(m n)=\operatorname{diag}\left\{d_{m n, 1}, d_{m n, 2}, \mathrm{~L}, d_{m n, K}\right\}$.

We can conduct eigen-value decomposition of $\mathbf{R}(m n)$, and get the signal subspace $\mathbf{E}_{s}$. Then there must be a unique, non-singular and full rank matrix $\mathbf{T} \in \square^{K \times K}$, which makes the Eq.(6) set up.

$\mathbf{E}_{s}=\left[\begin{array}{c}\mathbf{A}_{r x} \\ \mathbf{A}_{r x} \mathbf{\Psi} \\ \mathbf{M} \\ \mathbf{A}_{r x} \boldsymbol{\Psi}^{N}\end{array}\right] \mathbf{T}$

We partition $\mathbf{E}_{\mathrm{s}}$ as

$\mathbf{E}_{\mathrm{s}}=\left[\begin{array}{l}\mathbf{E}_{\mathrm{x}} \\ \mathbf{E}_{\mathrm{s} 1}\end{array}\right]=\left[\begin{array}{l}\mathbf{E}_{\mathrm{s} 2} \\ \mathbf{E}_{\mathrm{y}}\end{array}\right]$

where $\mathbf{E}_{\mathrm{s} 1}$ and $\mathbf{E}_{\mathrm{s} 2}$ represent the last $(M+1)$ rows and the first $(M+1)$ rows of matrix $\mathbf{E}_{\mathrm{s}}$ respectively, $\mathbf{E}_{\mathbf{x}}$ and $\mathbf{E}_{\mathbf{y}}$ are formed by the first $N(M+1)$ rows and the last $N(M+1)$ rows of $\mathbf{E}_{\mathrm{s}}$.

Then:

$\mathbf{E}_{\mathbf{y}}=\mathbf{E}_{\mathbf{x}} \mathbf{T}^{-1} \boldsymbol{\Psi} \mathbf{T}$

That is

$\mathbf{E}_{\mathbf{x}}^{+} \mathbf{E}_{\mathbf{y}}=\mathbf{T}^{-1} \boldsymbol{\Psi} \mathbf{T}$

therefore,

$\hat{u}_{k}^{i n i}=-\operatorname{angle}\left(\gamma_{k}\right) \lambda / 2 \pi d$

where $u_{k}=\sin \phi_{k} \sin \theta_{k}$ and $\gamma_{k}$ is the $k$ th eigen-value of $\mathbf{E}_{x}^{+} \mathbf{E}_{y}$.

Then, we replace $\mathbf{E}_{\mathrm{s}}$ to get

$\mathbf{E}_{\mathrm{s}}^{\prime}=\left[\begin{array}{c}\mathbf{A}_{r y} \\ \mathbf{A}_{r y} \boldsymbol{\Phi}_{x} \\ \mathbf{M} \\ \mathbf{A}_{r y} \boldsymbol{\Phi}_{x}^{M}\end{array}\right] \mathbf{T}^{\prime}$

where $\boldsymbol{\Phi}_{x}=\operatorname{diag}\left\{e^{-\mathrm{j} 2 \pi d \cos \phi_{1} \sin \theta_{1} / \lambda}, \mathrm{L}, e^{-\mathrm{j} 2 \pi d \cos \phi_{K} \sin \theta_{K} / \lambda}\right\}$ and $\mathbf{T}^{\prime}$ is permutation matrix. Similar to the above process, we can get

$\hat{v}_{k}=\cos \hat{\phi_{k}} \sin \hat{\theta}_{k}$

After pairing, we may obtain $\hat{\theta_{k}}$ and $\hat{\phi_{k}}$ by the following formula

$\hat{\theta}_{k}=\arcsin \sqrt{\hat{v}_{k}^{2}+\hat{u}_{k}^{2}}$

$\hat{\phi_{k}}=\arctan \left(\hat{v}_{k} / \hat{u}_{k}\right)$

The major complexity of ESPRIT algorithm via reconstructing Toeplitz-like matrix to estimate DOA of coherent signal is $O\left\{L(2 M+1)^{2}(2 N+1)^{2}+(M+1)^{3}(N+1)^{3}+2 K^{2}[M(N+1)+N(M+1)]+6 K^{3}\right\}$.

\section{Simulation results}

Define the root mean squared error (RMSE) as 
$R M S E=\frac{1}{T} \sum_{m=1}^{T} \sqrt{\frac{1}{K} \sum_{k=1}^{K}\left(\omega_{k m}-\omega_{k}\right)^{2}}$

where $T$ is simulation times, $\omega_{k m}$ represents the estimation value of the $k$ th source elevation (or azimuth) in the $m$ th simulation, $K$ is the number of coherent signal sources, and $\omega_{k}$ is the accurate value of the $k$ th source elevation (or azimuth). In the following simulations, we define $K$ is the source number, $L$ is the number of snapshots, and $(2 M+1) \times(2 N+1)$ is the number of array elements. To ensure the commonality, we set $N=3$.

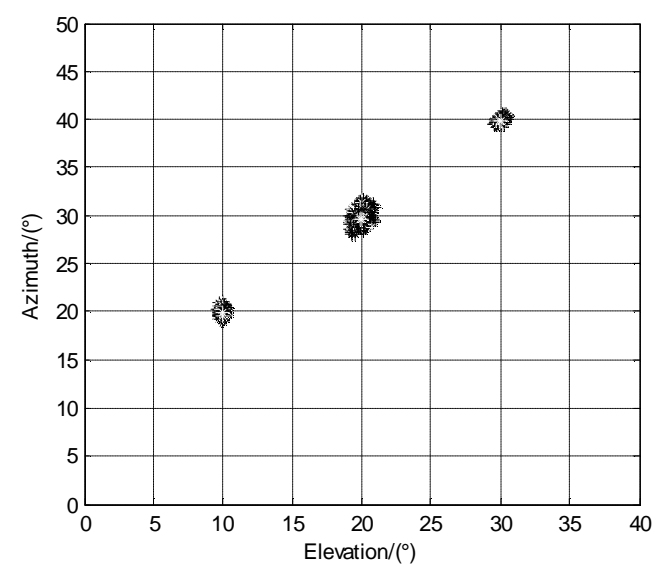

Fig.2 Angle estimation performance of the proposed algorithm with $\mathrm{SNR}=30 \mathrm{~dB}(L=100, M=3$ and $K=3$ )
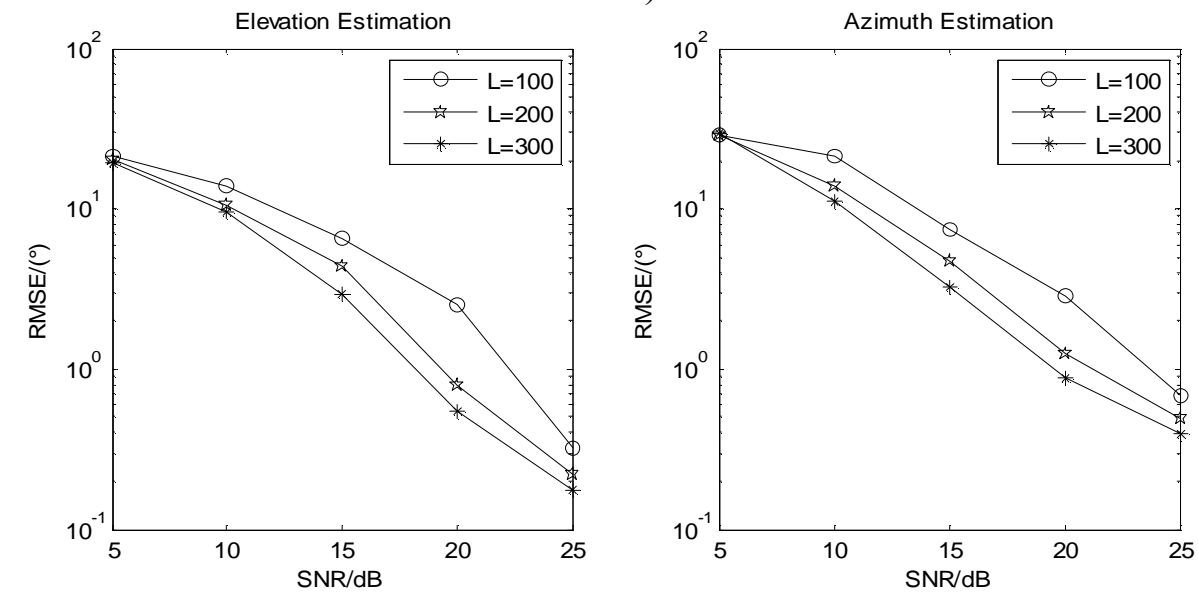

Fig. 3 Angle estimation performance with different $L(M=3$ and $K=3)$
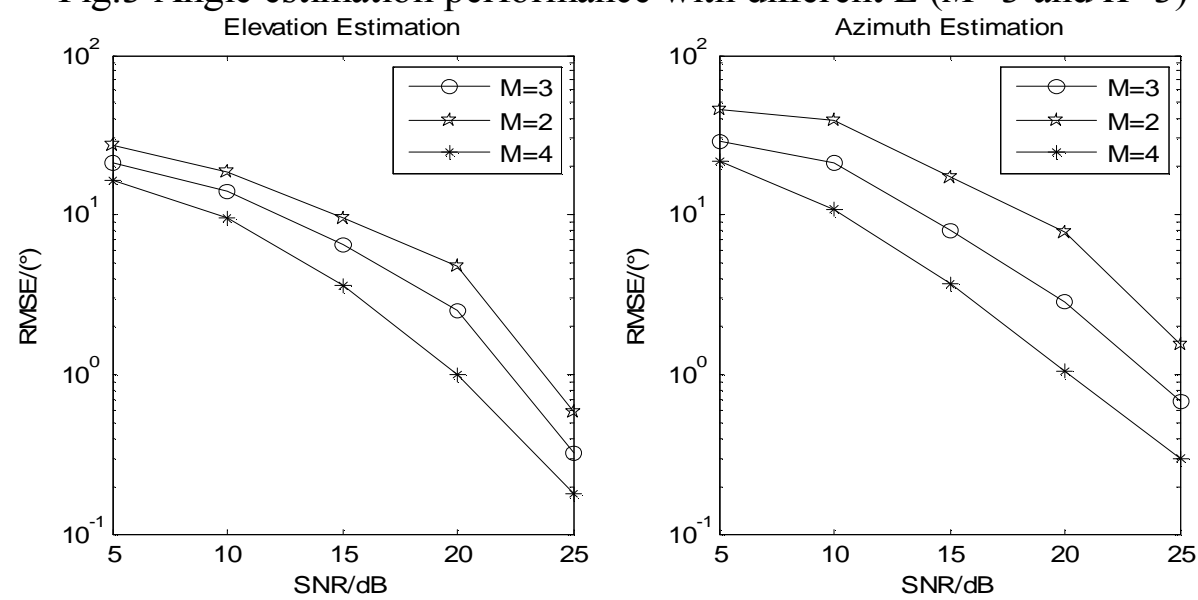

Fig.4 Angle estimation performance with different $M(L=100$ and $K=3)$ 

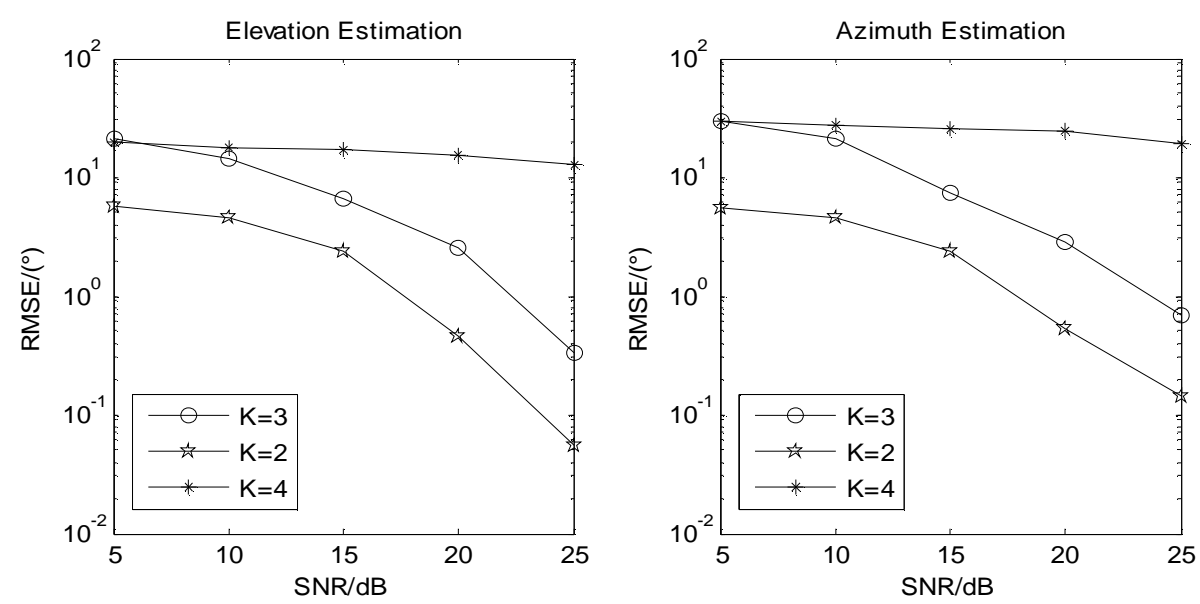

Fig.5 Angle estimation performance with different $K(L=100$ and $M=3)$

Fig. 2 depicts angle estimation performance of the proposed algorithm in $\mathrm{SNR}=30 \mathrm{~dB}$, where $M=3$, elevations are $\left(10^{\circ}, 20^{\circ}, 30^{\circ}\right)$, and azimuths are $\left(20^{\circ}, 30^{\circ}, 40^{\circ}\right)$. From the figure we can see that this algorithm estimate the elevation and azimuth effectively. Fig. 3 presents the angle estimation performance with different snapshots. As the snapshots increase, the sample data augments so that the covariance matrix becomes more accurate and the DOA estimation performance improves. Fig. 4 presents the angle estimation performance with different numbers of array elements. From Fig.4, we can find that the DOA estimation performance is improved with the increase in the number of the elements. Fig. 5 investigates angle estimation performance with different signal source quantities. We see from the Fig. 5 that the DOA estimation performance decreases as the signal sources increase.

\section{Conclusions}

The paper has presented the problem of two-dimension coherent DOA estimation for uniform planar array, and proposed the 2D coherent DOA via Toeplitz matrix and ESPRIT algorithm. The proposed algorithm reconstructs the Toeplitz matrix to eliminate the coherence, and use ESPRIT algorithm for 2D-DOA estimation. The proposed algorithm can obtain automatically paired 2D-DOA estimate, and it has a low computational complexity.

\section{References}

[1] Zhang X, Wang F, Chen W, et al. Theory and application of array signal processing[M]. Beijing: National Defense Industry Press, edition 2, 2013. (in Chinese)

[2] Kim J M, Lee O K, Ye J C. Compressive MUSIC: revisiting the link between compressive sensing and array signal processing[J]. IEEE Transactions on Information Theory, 2012, 58(1): 278-301.

[3] Naidu P S. Sensor array signal processing[M]. CRC press, 2010.

[4] Kundu D. Modified MUSIC algorithm for estimating DOA of signals[J]. Signal Processing, 1996, 48(95):85-90.

[5] Zoltowski M D, Haardt M, Mathews C P. Closed-form 2D angle estimation with Rectangular arrays in element space or beamspace via unitary ESPRIT [J]. IEEE Trans. Signal Process. , 1996, 44 (1): 326-328.

[6] Zhang X, Gao X, Chen W. Improved blind 2D-direction of arrival estimation with L-shaped array using shift invariance property[J]. Journal of Electromagnetic Waves and Applications, 2009, 23(5-6): 593-606.

[7] Marcos S, Marsal A, Benidir M. The propagator method for source bearing estimation[J]. Signal Processing, 1995, 42(2): 121-138. 
[8] Sanchez-Aranjo J, Marcos S. Statistical analysis of the propagator method for DOA estimation without eigendecomposition[C]. IEEE Signal Processing Workshop on Statistical Signal and Array Processing, 8th 1996: 570-573.

[9] Forster P, Larzabal P, Boyer E. Threshold performance analysis of maximum likelihood DOA estimation[J]. IEEE Transactions on Signal Processing, 2004, 52(11):3183 - 3191.

[10]Xiaofei Z, Jianfeng L, Lingyun X. Novel two-dimensional DOA estimation with L-shaped array[J]. EURASIP Journal on Advances in Signal Processing, 2011, 2011(1): 1-7.

[11]Chen, Y.-H, Lin, et al. Fourth-order cumulant matrices for DOA estimation[J]. IEE Proceedings Radar Sonar and Navigation, 1994, 141(3):144-148.

[12]Dai J, Ye Z. Spatial Smoothing For Direction Of Arrival Estimation Of Coherent Signals In The Presence Of Unknown Mutual Coupling[J]. Signal Processing, IET, 2011, 5(4):418 - 425.

[13] Gu Z, Gunawan E. A performance analysis of multipath direction finding with temporal smoothing[J]. Signal Processing Letters, IEEE, 2003, 10(7):200 - 203.

[14]Han F M, Zhang X D. An ESPRIT-like algorithm for coherent DOA estimation[J]. IEEE Antennas \& Wireless Propagation Letters, 2005, 4:443 - 446. 